\title{
Analysis of the factors influencing on bird nesting and its impact on railway operation
}

\author{
Lijuan Shi, Zuoning Jia, Huize Sun and Mingshu Tian \\ Tongji University, Shanghai, China, and \\ Liquan Chen \\ China Railway Guangzhou Group Co., Ltd., Guangzhou, China
}

Influencing on bird nesting

\begin{abstract}
Purpose - This paper aims to study the affecting factors on bird nesting on electronic railway catenary lines and the impact of bird nesting events on railway operation.

Design/methodology/approach - First, with one year's bird nest events in the form of unstructured natural language collected from Shanghai Railway Bureau, the records were structured with the help of python software tool. Second, the method of root cause analysis (RCA) was used to identify all the possible influencing factors which are inclined to affect the probability of bird nesting. Third, the possible factors then were classified into two categories to meet subsequent analysis separately, category one was outside factors (i.e. geographic conditions related factors), the other was inside factors (i.e. railway related factors).
\end{abstract}

Findings - It was observed that factors of city population, geographic position affect nesting observably. Then it was demonstrated that both location and nesting on equipment part have no correlation with delay, while railway type had a significant but low correlation with delay.

Originality/value - This paper discloses the principle of impacts of nest events on railway operation.

Keywords Risk management, Bird nest, Geographic information, Lognormal stochastic model, RAC analysis, Railway operation, Statistic analysis

Paper type Research paper

\section{Introduction}

With the development of high-speed railway, it plays an increasingly important role in national transportation system. The safety and efficiency of high-speed train operation is getting seriously concerned. Short-circuit trip of railway catenary lines caused by bird nesting, which is a very common kind of risk event, generally exerts a significant negative impact on train operation and ultimately decreases the efficiency and safety of railway transport.

(C) Lijuan Shi, Zuoning Jia, Huize Sun, Mingshu Tian and Liquan Chen. Published in Smart and Resilient Transportation. Published by Emerald Publishing Limited. This article is published under the Creative Commons Attribution (CC BY 4.0) licence. Anyone may reproduce, distribute, translate and create derivative works of this article (for both commercial and non-commercial purposes), subject to full attribution to the original publication and authors. The full terms of this licence may be seen at http://creativecommons.org/licences/by/4.0/legalcode

Authors would like to acknowledge the support of the research program of Comprehensive Support Technology for Railway Network Operation (2018YFB1201403), which is a subproject of Advanced Railway Transportation Special Project belonging to the 13th Five-Year National Key Research and Development Plan funded by Ministry of Science and Technology of China. 
SRT

2,1

The risk event caused by bird nesting is next to lightning strike and external force destruction, ranking the third in the total number of power supply line faults. According to the investigation and statistical analysis of various types of catenary bird damage faults, the most prominent bird damage fault is nesting, which is called nest event in this paper. The specific parts of the catenary lines equipment of electrified railway are very suitable for birds nesting, such as supporting column, hard horizontal beam and other positions. Due to the selection of a variety of bird nesting materials, including steel wire and wooden rods, etc., nesting in these locations is easy to cause a short circuit, trip-off and other faults in the railway power supply line, affecting the normal railway transport order and even damage the equipment. Since 2010, 1,671 nests have been treated in one power supply section, and in 2011 alone, 516 nest locations were found and treated in a section of the Wenzhou-Fuzhou and Fuzhou-Xiamen high-speed railway, causing five trip-offs and affecting power supply for up to $1 \mathrm{~h}$ and $12 \mathrm{~min}$ at a time (Wang, 2013).

There are few studies on the risk events caused by bird nesting and the impacts of nest events on train operation quantitatively (Lin, 2016; Boqing et al., 2017). Generally, empirical judgment was applied in practice.

Duan Wangwang proposed a method of HOG feature-based detection of the nests. The method was adopted that first roughly observing, then fining the detection process of nesting, and finally determining the bird's nest area through probability density estimation (Duan, 2016). Li Tengfei analyzed the shortage of the traditional bird-driving method in monitoring and management and studied the relationship between structure and nest phenomenon of the catenary device, and his work put forward further of a catenary bird-driving system developed on the detection, communication, data processing and other function, providing a reference for further research and development of the similar system $(\mathrm{Li}, 2017)$.

Wang Mengyang, Lin Jiansheng, Wang Rui and many other researchers drew conclusions on the habit of birds and influenced factors of the nesting behaviors thorough analysis in terms of both time and space dimensions, including topography and geomorphology, time characteristics, location characteristics, repeated features and material characteristics, which to some extents inspires the catalog of characteristics in this paper (Lin, 2016; Boqing et al., 2017; Wang, 2018; Wang et al., 2008).

In the following sections of this paper, first, with the obtained one year's risk events in the form of natural language from Shanghai region railways, the nest event related records were selected and possible affecting factors were extracted. Second, all the possible factors which are inclined to bird nesting were identified with the method of root cause analysis (RCA). Then, with the help of SPSS, ArcGIS and other statistic methods, analysis the correlation of outside factors (geographic characteristics factors data from the internet) and inside factors (railway related factors data from Shanghai Railway Bureau) with bird nesting events, respectively. Finally, model the effects of nest events on railway operation.

\section{Data collection and pre-processing}

The data collected in the study were from two sources, one is from Shanghai Railway Bureau and the other is geographic data collected from the internet.

There are more than 2,500 railway event records formed in natural language obtained from Shanghai region railway network during almost one year, among which the nest events were selected and converted into structured data partly with the help of Python software modules such as XLRD and XLWT. There are 275 structured nest event records with all possible valid attribute information for further analysis, such as nesting location (station or section), bird nesting event time, nesting on equipment part of catenary lines, train type and the delay of the nest events on train operation. 
As to the geographic data, it is observed that temperature, vegetation coverage, population, geographic position (longitude and latitude) and nearby water, etc., may influence bird nesting. Every location of a bird nest event from each of the 275 records was mapped to a corresponding railway station or a section between two adjacent stations. The cumulative frequency distribution of bird nesting events at stations or sections along the railway lines was obtained for further relevant environmental factors analysis from a geographic perspective. The APIs from the map's POI were used to search these geographically relevant data along the studied railway lines. Besides, high-resolution raster data such as tree distribution map of China, population density map of the sixth population census and water system map of China are collected from major research websites for further analysis.

\section{Analysis of the possible influencing factors on nesting with root cause analysis method}

$\mathrm{RCA}$ is a structured problem-solving method, which is used to gradually find out the causes of the problem so that the problem can be solved effectively and pertinently. The approach of RCA consists of seven steps: selecting team members, actions to prevent the problem from spreading, identifying root causes, further solutions, implementing solutions and evaluating performance. The root cause identification step is the key of RCA method. During the process of nesting factors analysis, we focused more on the steps of identifying root causes and further solutions. In the RCA stage, RCA method provides four RCA tools, including causality diagram, brainstorming method, fishbone diagram and why-why diagram (Xu, 2006).

The fishbone diagram was chosen to help the analysis of the possible influencing factors on nesting. The application process of fishbone diagram was as follows:

- state the problem of bird nesting;

- identify major cause categories. According to the difference in data sources and analysis methods, two categories are decided, i.e. railway related factors and geographic characters;

- use the brainstorm method to find the reasons under each category, including nesting time, nesting on the equipment part, railway type, the delay of the nest events on train operation, nesting location (station or section), geographic position and environmental factors;

- Consider the factors that may contribute to each cause and put them on a line from the cause. For instance, environmental cause can be divided into water distribution, population and vegetation coverage factors. So those lines were drawn from environment to the coverage factors;

- discuss how the factors affect bird nesting; and

- discuss the way to prove the causes and factors using data analysis.

According to the collected data, the factors probably affecting bird nesting were depicted in the fishbone diagram as shown in Figure 1.

For the sake of the convenience of expression, the factors extracted from the nest event records are given as the general term "inside factors", including nesting location (station, section), event time (discovery time, responding time, end time), nesting on equipment parts of catenary lines (supporting column, hard horizontal beam, compensation device, pedestal, protecting mesh, disconnector switch, return line shoulder, insulator, etc.), railway type
Influencing on bird nesting

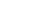




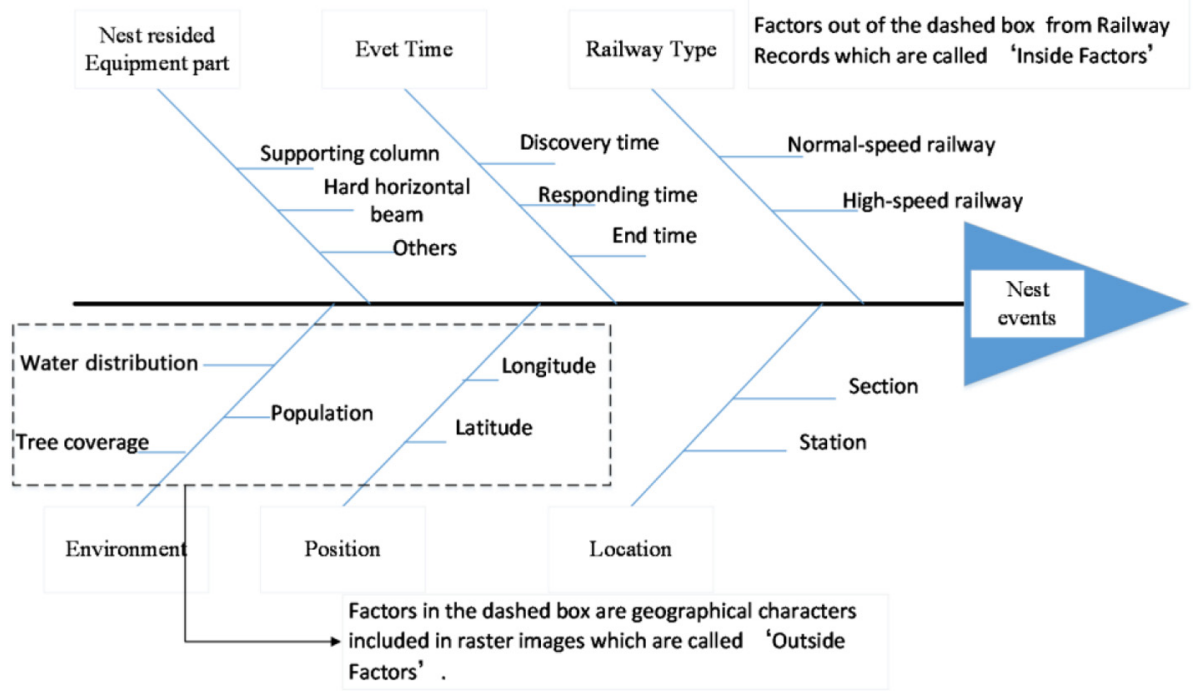

Figure 1.

Fishbone diagram

(normal-speed railway, high-speed railway), the delay of the nest events on train operation, etc. The factors obtained from the geographic data is named as "outside factors", such as temperature, vegetation coverage, population, longitude and latitude (geographic position) of an event position and the distribution of the surrounding water.

\section{Analysis of "outside factors" affecting on probability of bird nesting}

The accumulated nest event number of every railway station positioned by latitude and longitude is imported into the data visualization map platform via APIs. A hexagonal heat map of bird nest events along the railways was obtained and as shown in Figure 2.

It can be obviously observed from Figure 2, bird nest events occurred most frequently on three sections of railway lines, i.e. Shanghai-Hangzhou line, the Nanjing-Shanghai section of the Beijing-Shanghai line and the Xuzhou-Lianyungang section of the Longhai line. It seems that the frequency of bird nest events is closely related to the population density of cities, geographic conditions such as water distribution.

To disclose whether "outside factors" do affect the frequency of bird nesting events or not, further study was conducted. First, every position of each nesting event record was marked on the map, which was expressed in the form of points on the map. Then, "outside factors" including the population density, vegetation coverage and water distribution in forms of raster data surrounding the positions of nesting events were collected. Buffers were established with Arcgis software which covered the studied geographic areas, as shown in Figure 3. Every event point is taken as the center of three circles with $10 \mathrm{~km}, 20 \mathrm{~km}$ and $50 \mathrm{~km}$ radius separately, which consist of three kinds of buffers with $20 \mathrm{~km}, 40 \mathrm{~km}$ and $100 \mathrm{~km}$ in width for all event points.

With Arcgis statistics tools, vegetation coverage, population density and water distribution data within the buffers are calculated for analysis. The visual results are events surrounding buffers with $10 / 20 / 50 \mathrm{~km}$ circle radius, vegetation coverage, population density and water distribution, respectively, as shown in Figures 3-6. For example, in Figure 3, the base map is China population distribution map, on which drawn the yellow, green and blue circles with $10 \mathrm{~km}, 20 \mathrm{~km}$ and $50 \mathrm{~km}$ radius, respectively. The summation of population is calculated in each circle. 


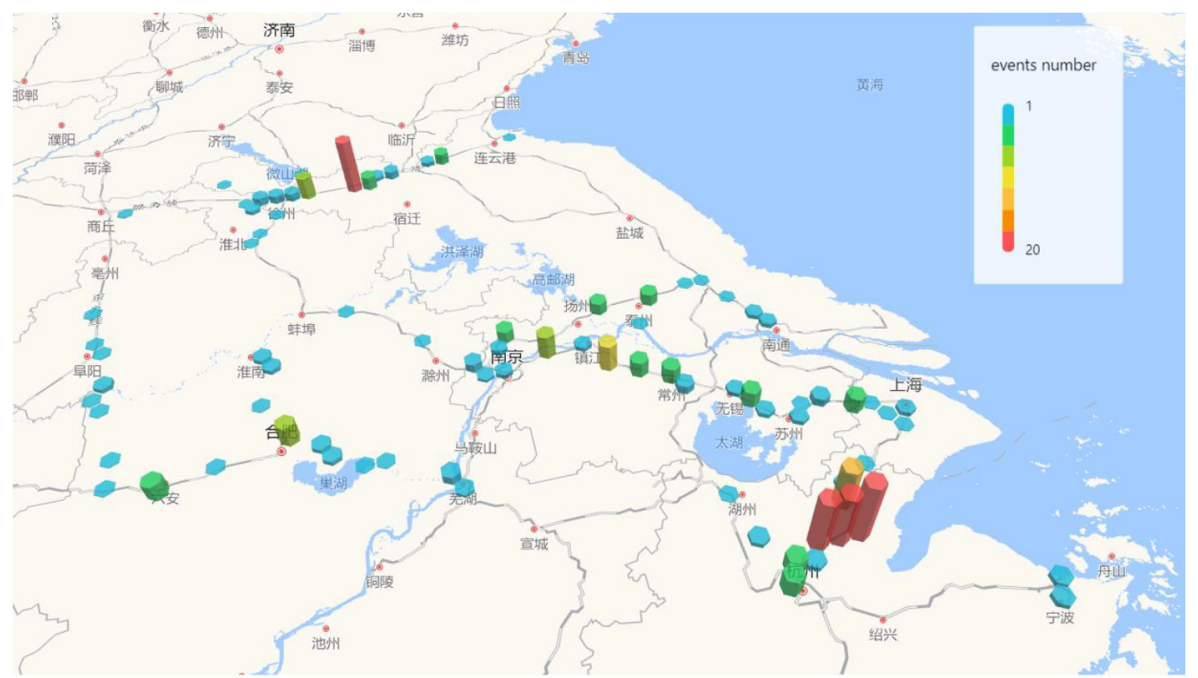

Source: https://maplab.amap.com/share/mapv/0b93826ea0e9a033118122c416f78d57

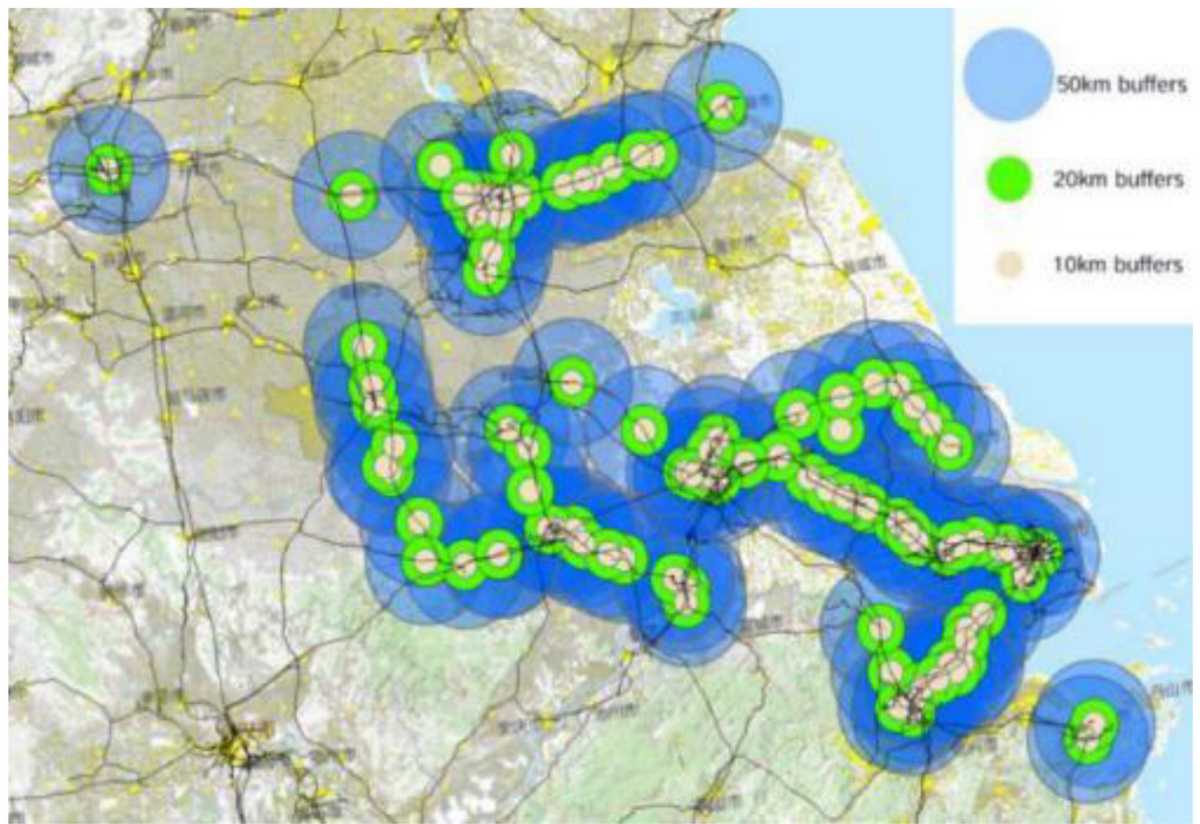

Influencing on bird nesting

27

Figure 2.

A heat map of bird nest events along the railways
Figure 3.

Buffers with $10 / 20 / 50 \mathrm{~km}$ radius

In Figures 3-6, some characteristics of these possible affecting "outside factors" could be learned intuitively. Nest events occurred in areas with sparse tree distribution, which is different from the previous studies. Nest events tend to occur in densely populated areas. Nest events are prone to occur near the water areas. 
SRT

2,1

28

Figure 4.

Vegetation coverage
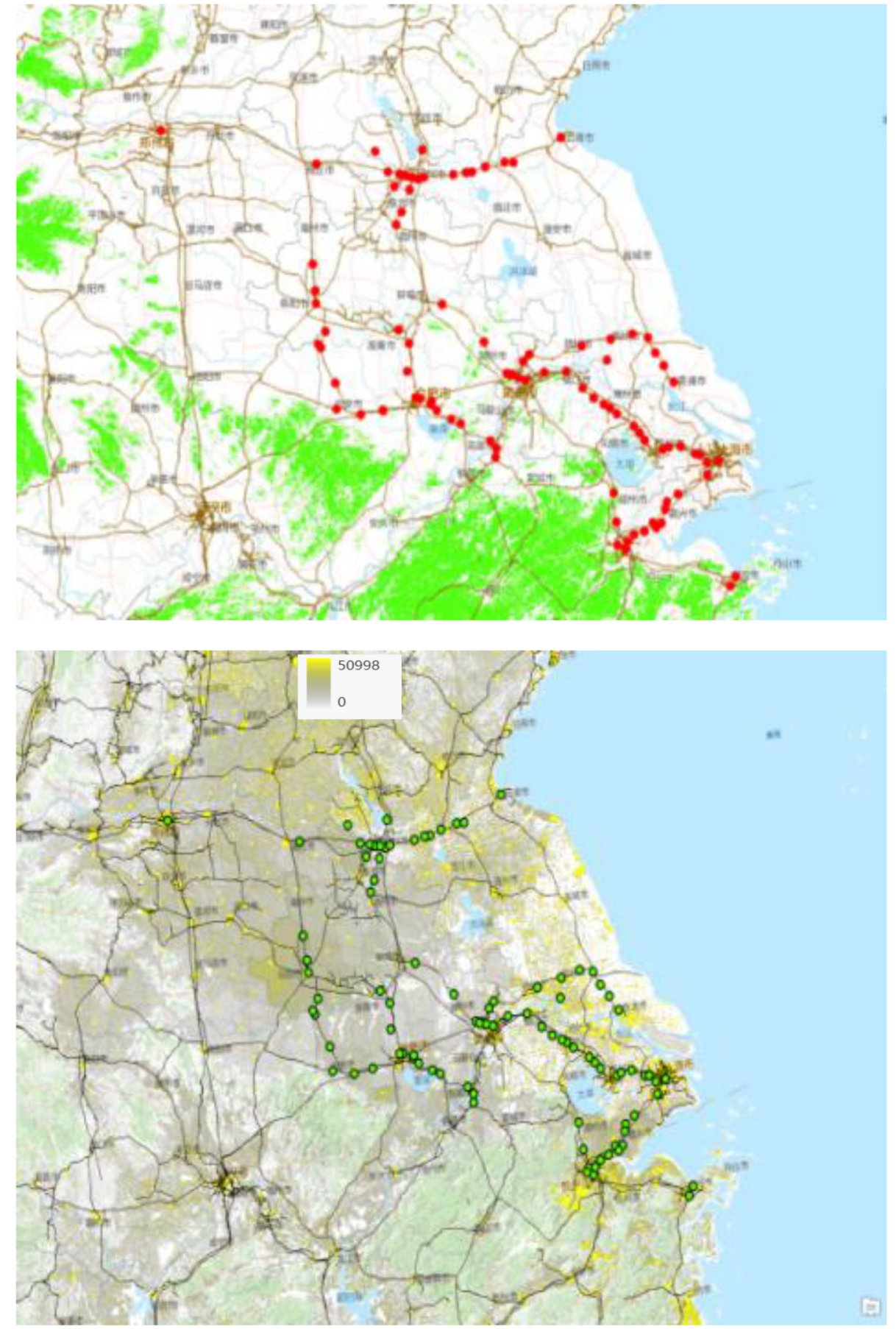

Figure 5.

Population density 
Apparently, nest events are correlated with surrounding factors such as vegetation coverage, population density, distance from water areas, then statistical data analysis in SPSS was done to get the scientifically rigorous conclusions.

The following conclusions come out after the analysis of the correlation between the above data and the frequency of bird nest events. As demonstrated in Table 1, there is no correlation between the nest event frequency and vegetation coverage. The correlation between the nest event frequency and population density is shown in Table 2 . It makes an obvious point that the higher population density, the higher nest event frequency. The correlation between the event frequency and water distribution of station is shown in Table 3. It illustrates that water distribution is positively related to nest event frequency in spring.

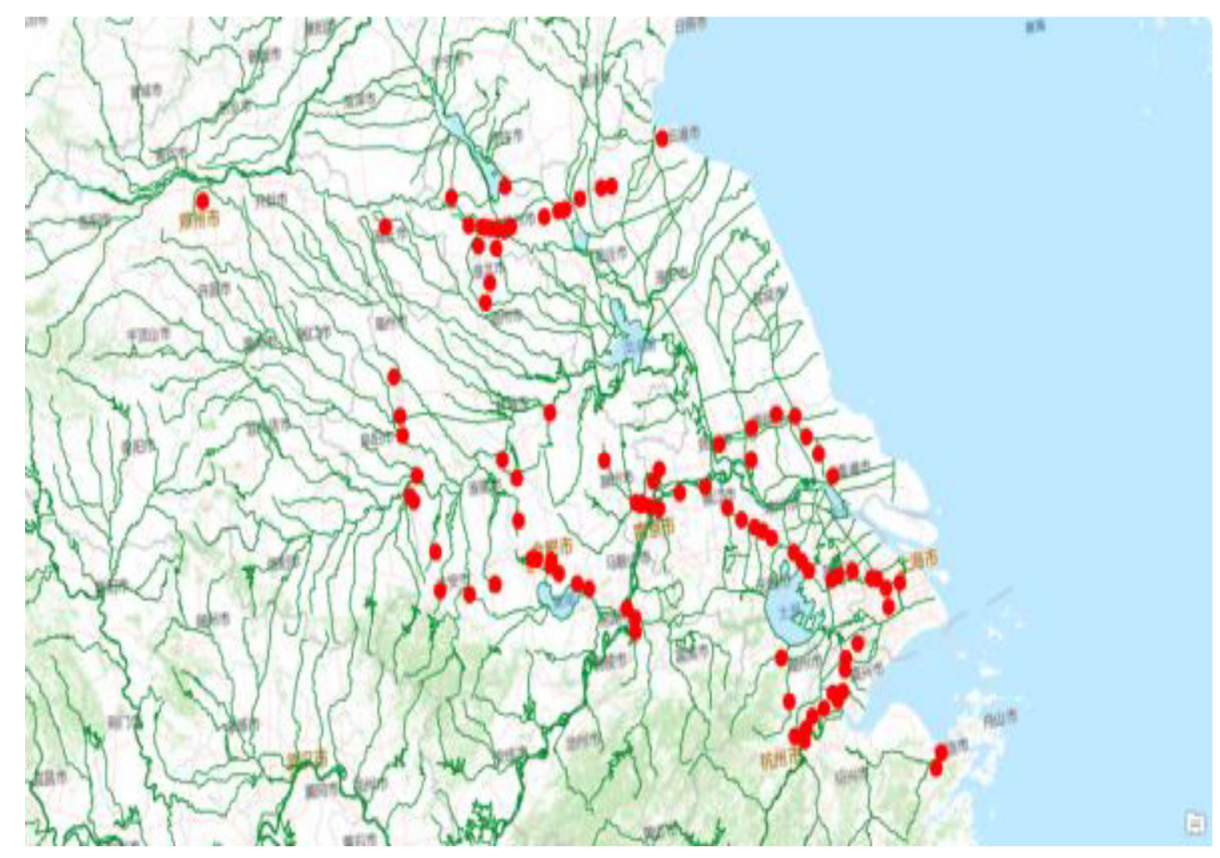

Correlation coefficient

$10 \mathrm{~km}$ radius

$20 \mathrm{~km}$ radius

$50 \mathrm{~km}$ radius

Spearman

Significance level

0.051

0.01

0.022

0.01

0.121

0.01
Influencing on bird nesting

Figure 6.

Water distribution

Table 1.

The correlation between the nest event frequency and vegetation coverage

\begin{tabular}{lccc}
\hline Correlation coefficient & $10 \mathrm{~km}$ radius & $20 \mathrm{~km}$ radius & $50 \mathrm{~km}$ radius \\
\hline Spearman & 0.272 & 0.272 & 0.251 \\
Significance level & 0.01 & 0.01 & 0.05 \\
\hline
\end{tabular}

Table 2.

The correlation between the nest event frequency and population density 
SRT

2,1

30

Table 3.

The correlation between the nest event frequency and water distribution

\section{Characteristics of "inside factors" on nesting events}

\subsection{Primary analysis of nest events}

Based on above analysis, the obtained preprocessed 275 structured nest event records include multidimensional parameters, such as time, location, railway type and nest resided equipment part. Each parameter has a number of different values.

The statistical result of the 275 records indicates that the nest events Spatio-temporal distribution characteristics in proportion as follows, as shown in Figure 7:

- The nest events generally happen in spring, covering the period from January to early April and $92 \%$ of nest events occurred in February and March. Bird nesting event accounts for a third of all railway risk events in spring.

- $71 \%$ of the nest events took place in the sections, while $29 \%$ in stations.

- $13 \%$ nest events took place in high-speed railways, while $87 \%$ took place in normalspeed railways.

- As to birds' favorite nesting equipment part, $73 \%$ bird nests resided on supporting column of catenary, while $20 \%$ on hard horizontal beam and sum of other cases accounts for $7 \%$.

\subsection{Correlation analysis of "inside factors" on event lasting time}

Bird nesting event lasting time (hereinafter, also called delay) is given by event end time minus discovery time, which is important to determine the severity of impact of nesting event on train operation. All the possible inside factors are enumerated to conduct the

\begin{tabular}{cccc}
\hline & Correlation coefficient & $p$-value & Significance level \\
\hline Spearman & 0.211 & 0.036 & 0.05 \\
\hline
\end{tabular}

\section{Figure 7.}

The nest events Spatio-temporal distribution characteristics

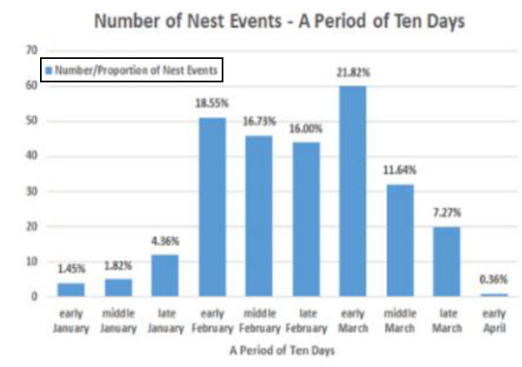

Location

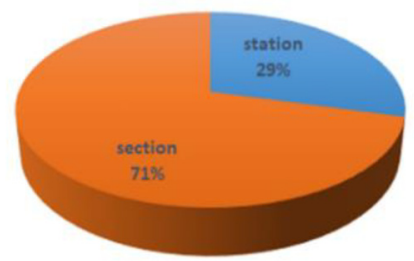

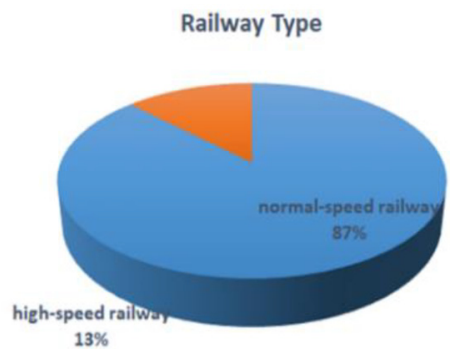

Nest Resided Equipment Part

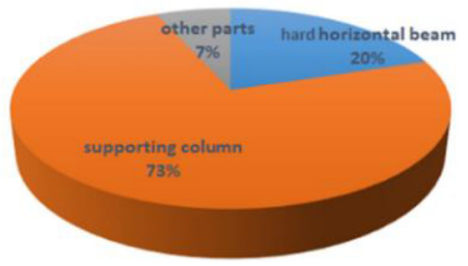


correlation analysis with event lasting time to determine the significant influence factors. The explanation for the possible inside factors is as follows:

- Location. The event took place in station or section which is represented by 0 or 1 in correlation analysis.

- Railway type. The event happened on normal-speed railway or high-speed railway which is denoted by 0 or 1 , respectively.

- Nesting on equipment part. There are mainly nine different parts birds generally nest on the catenary lines, including supporting column, hard horizontal beam, compensation device, pedestal, protecting mesh, disconnector switch, return line shoulder, insulator, which are expressed as 1 to 9 , respectively, in the correlation analysis.

Correlation analysis of inside factors and event lasting time (i.e. delay) is conducted, as shown in Table 4. There are two values for each pair of correlation analysis. The first value in upper cell is the Spearman correlation coefficient. The second value in lower cell is the $p$ value, $\alpha=5 \%$. Italic Figures are statistically insignificant. It is observed that both location and nesting on equipment part have no correlation with delay, while railway type has a significant but low correlation with delay. The Spearman correlation coefficient was 0.294.

\section{Delay stochastic model of different railway type}

\subsection{Delay frequency distribution}

Data of all records were preprocessed by mathematical statistics method. The statistical delays caused by bird nesting events with more than three times of variance added to the mean were removed as abnormal data. The remaining 233 normal-speed railway event records and 34 high-speed railway event records are statistically analyzed in the following.

Summary statistics for delay of different railway types are shown in Table 5, and delay frequency distributions of normal-speed railway and high-speed railway are shown in Figure 8, respectively.

\subsection{Delay stochastic model fit}

From the visual inspection of Figure 8, two stochastic models seem plausible: Lognormal and Weibull. Consequently, two models are proposed in this section to fit the delay frequency distributions of two type railways, respectively.

Influencing on bird nesting

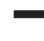


SRT

2,1

Figure 8.

Frequency distributions for delay of different railway types

The results of delay frequency distributions fit shown in Table 6 are measured by the squares sum of error (SSE) and $R^{2}$. The SSE in the square of the difference between the actual value and the fitted value. The fit is more realistic when the SSE is smaller and the $R^{2}$ is correspondingly closer to 1 .

Thus, we could tell from Table 6 that the Lognormal stochastic model is the best-fit model for delays caused by bird nesting events of both normal-speed railway and highspeed railway. $\mathrm{K}-\mathrm{S}$ test is then applied for hypothesis testing the Lognormal models of the two railway types. The results imply that delays under each railway type are lognormally distributed at the $5 \%$ significance level.

\subsection{Proposed model}

Based on above analysis, Lognormal stochastic model is the proposed model for the delay frequency distribution under two railway types. The delay Lognormal probability density functions of normal-speed railway and high-speed railway, respectively, are shown in Table 7. Then the corresponding probability density and cumulative probability distributions are depicted in Figure 9, which are used for evaluating the delay probability when a bird nesting event happens on normal-speed railway or high-speed railway. $t_{0.9}$ means $90 \%$ of delays occur

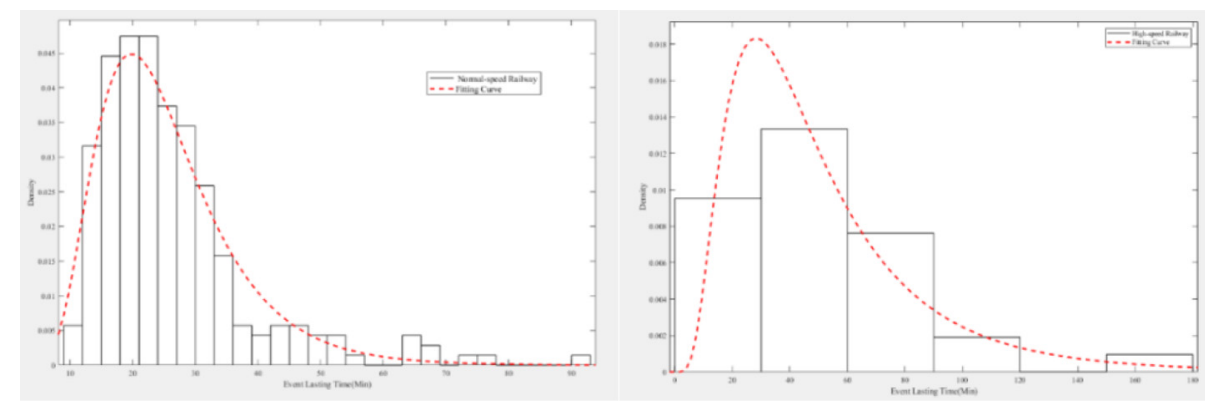

\section{Table 6.}

Delay frequency distributions fit under different railway types

\begin{tabular}{lllc}
\hline Distribution fitting & Stochastic model & \multicolumn{1}{c}{ SSE } & $R^{2}$ \\
\hline Normal-speed railway & Lognormal & 0.00026294 & 0.9637 \\
& Weibull & 0.0015 & 0.7916 \\
High-speed railway & Lognormal & 0.00002848 & 0.8420 \\
& Weibull & 0.00005367 & 0.7022 \\
\hline
\end{tabular}

\section{Table 7 .}

The fitted delay lognormal probability density functions of two railway types

\begin{tabular}{llcc}
\hline Railway type & Lognormal probability density functions & $\mathrm{t}_{0.9}(\mathrm{~min})$ & $\mathrm{t}_{0.95}(\mathrm{~min})$ \\
\hline Normal-speed railway & $f(t)=\frac{1}{0.4249 \times t \sqrt{2 \pi}} e^{-\frac{1}{2}\left(\frac{\ln t-3.163}{0.4249}\right)^{2}}$ & 41 & 48 \\
High-speed railway & $f(t)=\frac{1}{0.6319 \times t \sqrt{2 \pi}} e^{-\frac{1}{2}\left(\frac{\ln t-3.739}{0.6319}\right)^{2}}$ & 95 & 119 \\
\hline
\end{tabular}



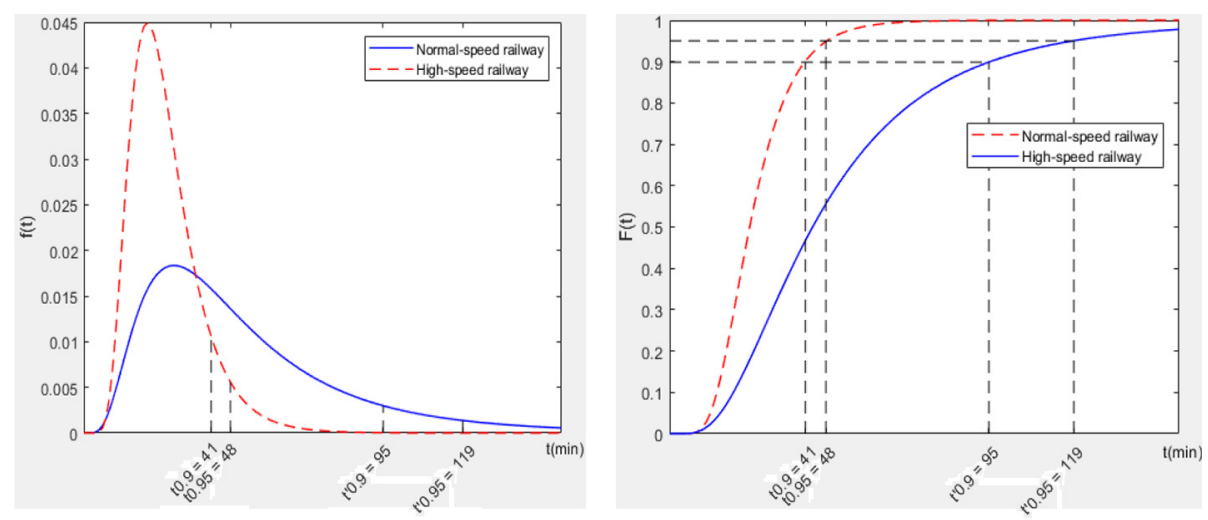

Influencing on bird nesting

33

Figure 9.

Probability density and cumulative probability distributions

within $t_{0.9}$ time, and so does for $t_{0.95}$. The results also indicate that $90 \%$ chance of a delay caused by a bird nest event is less than $41 \mathrm{~min}$ for normal-speed railway and less than $95 \mathrm{~min}$ for high-speed railway.

\section{Conclusion}

This paper analyzed all the possible factors influencing on bird nesting on railway catenary lines, with obtained one year's Shanghai region railway operation events data from Shanghai Railway Bureau and geographic data from the internet, to find out which factors had a statistically significant impact on probability of bird nesting and the characteristics of nesting events. Then, delay stochastic models were built to illustrate the influence quantitatively of the bird nesting events on the delay of railway operation for normal-speed railway and high-speed railway, respectively.

The findings are as follows:

- RCA method was used to analyze the possible influencing factors on bird nesting and a fishbone diagram is constructed to disclose all possible "inside factors" and "outside factors" affecting bird nesting on railway.

- As to the geographic factors, both population density and water distribution in spring as "outside factors" have positively significant influence on the frequency of nest events.

- The bird nesting events of Shanghai Bureau regional railway generally happen in spring. Most of the nest events took place in the sections of railway other than in stations. Supporting column of catenary is the birds' favorite nesting equipment.

- Lognormal stochastic models are the best-fit models for delays caused by bird nesting events of both normal-speed railway and high-speed railway, which are used for evaluating the delay probability when a bird nesting event happens on normal-speed railway or high-speed railway. Models disclose the principle of impacts of nest events on railway operation.

The findings are helpful to improve daily railway operation work in practice, for example, the patrol of bird nests should be strengthened in spring and in metropolitan areas with abundant water. The supporting column of catenary should be inspected particularly as an important part. Once a bird nesting event occurs on normal-speed railway or high-speed 
SRT

2,1

railway, pro-active measures can be taken in time to mitigate the event impact of railway operation based on the forecasted delay time.

\section{References}

Boqing, F., Li, P. and Yang, L. (2017), “Analysis of bird's nest disease of high-speed railway contact network based on spatio-temporal big data", China Railway, Vol. 1, pp. 29-34.

Duan, W. (2016), Detection and Abnormal Recognition of Catenary Equipment for Monitoring Video (in Chinese), Southwest Jiaotong University.

Li, T. (2017), Research and Development of Distributed Bird Driving System for Railway Catenary Based on Wireless Communication, Hefei University of Technology.

Lin, J. (2016), "Research on the prevention and control measures of bird pests in high-speed rail catenary", Straits Science, Vol. 8, pp. 24-27.

Wang, P. (2013), "Discussion on using sliding ladder under the condition of catenary fault", China Venture Capital, Vol. 250.

Wang, M. (2018), "Discussion on anti-bird measures of high speed railway catenary", Telecom Power Technologies, Vol. 7, pp. 237-238.

Wang, R., Yu, S. and Gao, L. (2008), "Fault analysis and prevention of bird damage on 220kV line", Progress in Power Grids and Hydropower.

$\mathrm{Xu}, \mathrm{D}$. (2006), "RCA/CA: root cause analysis and corrective action", The Science Education Collects, Vol. 4, pp. 185-186.

Corresponding author

Zuoning Jia can be contacted at:674767670@qq.com

For instructions on how to order reprints of this article, please visit our website: 